\title{
Expectativa x Experiência: análise de avaliações publicadas em redes sociais sobre a qualidade dos serviços de meios de hospedagem classificados pelo SBClass
}

\author{
Joelma Abrantes Guedes Temoteo ${ }^{a}$ \\ Jammilly Mikaela Fagundes Brandão ${ }^{b}$ \\ Jefferson Oliveira da Silva Lacerdac
}

\begin{abstract}
Resumo
O cliente, ao ver uma placa fixada na entrada de um hotel apresentando determinado número de estrelas, sente-se seguro de que a infraestrutura e a qualidade dos serviços prestados foram auditadas e o empreendimento atende a vários parâmetros de qualidade, gerando uma expectativa compatível com a categoria observada. No entanto, nem sempre a superação dessas expectativas geradas nos hóspedes e a satisfação total de suas necessidades são atingidas. Partindo de tal perspectiva, o objetivo central desta pesquisa consiste em analisar a percepção de hóspedes sobre a qualidade dos serviços de meios de hospedagem, classificados pelo SBClass nas categorias de cinco e quatro estrelas a partir de avaliações divulgadas no TripAdvisor e no Booking.com. Para tanto, buscou-se identificar quais hotéis da região Nordeste foram classificados pelo SBClass nas categorias mencionadas, e analisar, de forma comparativa, as notas atribuídas a esses empreendimentos e os comentários divulgados nas referidas redes sociais. Para atender aos objetivos estabelecidos, realizou-se uma pesquisa descritiva de abordagem qualitativa. Dentre as principais constatações, destaca-se que não foi verificada diferença relevante entre as notas recebidas pelos sete meios de hospedagem analisados. Também se observou que as notas gerais do TripAdvisor para as empresas hoteleiras são maiores do que as notas gerais atribuídas pelos clientes do Booking.com.
\end{abstract}

Palavras-chave: Qualidade; Redes sociais; Hotelaria; SBClass.

\section{Abstract}

Expectation $x$ experience: analysis of reviews published on social networks about the quality of hosting services classified by SBClass

A customer, after seeing a fixed sign at the entrance of a hotel with a certain number of stars, feels safe that the infrastructure and the quality of the services provided were audited and that the enterprise meets the various quality parameters, creating an expectation compatible with the observed category. However, not always the guests' expectations and the total satisfaction of their necessities are overcome. From such a perspective, the main objective of this research is to analyze the guest perception about the quality of hosting services, classified by SBClass in the categories of five and four stars based on

a. Doutoranda em Desenvolvimento e Meio Ambiente pelo Programa Regional de Pós-Graduação em Desenvolvimento e Meio Ambiente da Universidade Federal da Paraíba (UFPB). João Pessoa, Paraíba, Brasil. E-mail: joelma.abrantes@gmail.com

b. Doutoranda em Administração, pelo Programa de Pós-Graduação em Administração da UFPB. João Pessoa, Paraíba, Brasil. E-mail: jammillybrandao@gmail.com

c. Doutorando em Administração, pelo Centro de Pós-Graduação e Pesquisas em Administração da Universidade Federal de Minas Gerais (UFMG). Belo Horizonte, Minas Gerais, Brasil. E-mail: jeffersoncantalice3@gmail.com 
reviews published on TripAdvisor and on Booking.com. To this end, we identified which hotels of the Northeast region were classified by SBClass in the categories mentioned, and analyzed, by comparison, the ratings assigned to those enterprises and the comments disclosed on social networks. To meet the goals set, a descriptive research of qualitative approach was conducted. One of the main findings is that no relevant difference was observed among the ratings received by the seven hosting services analyzed. We also noted that the general ratings for the hotel companies on TripAdvisor are higher than the general ratings on Booking.com.

Keywords: Quality; Social networks; Hotel business; SBClass.

\section{Resumen}

\section{Expectativa $x$ experiencia: análisis de evaluaciones publicadas en las redes sociales acerca de la calidad de los servicios de hospedaje clasificados por SBClass}

El cliente, al ver una placa fijada en la entrada de un hotel que ofrece un número de estrellas, siente que la infraestructura y la calidad de los servicios han sido auditadas y el proyecto cumple con diversos parámetros de calidad, generando una expectativa en consonancia con la categoría observada. Sin embargo, ni siempre se puede superar las expectativas generadas en los huéspedes y satisfacer enteramente sus necesidades. Desde esta perspectiva, el objetivo central de esta investigación es analizar la percepción de los clientes sobre la calidad de los servicios de establecimientos hoteleros clasificados por SBClass en las categorías de 5 y 4 estrellas desde los comentarios publicados en TripAdvisor y Booking.com.Con este propósito, hemos tratado de identificar los hoteles en la Región Nordeste del Brasil que fueron clasificados por SBClass y analizar, de manera comparativa, las notas asignadas a estos establecimientos y las comentarios recogidos en las redes sociales. Para cumplir con los objetivos planteados, se realizó una investigación de enfoque cualitativo descriptivo. Entre las principales conclusiones, se destaca que no se observó diferencia significativa entre las notas recibidas por los siete establecimientos hoteleros analizados. También se observó que las notas generales asignadas a los hoteles en TripAdvisor son más altas que las puntuaciones globales concedidas por los clientes en Booking.com.

Palabras clave: Calidad; Redes sociales; Hospitalidad; SBClass.

\section{INTRODUÇÃO}

É comum associar a qualidade dos serviços de um meio de hospedagem ao número de estrelas que ele apresenta em sua fachada, ou mesmo em seus materiais de divulgação. No entanto, nem sempre a infraestrutura (equipamentos, instalações) e os serviços prestados atendem às expectativas geradas nos hóspedes e condizem com o que é divulgado. O Sistema Brasileiro de Classificação dos Meios de Hospedagem (SBClass) ${ }^{1}$, criado em 2011 pelo Ministério do Turismo (MTur), tem como objetivo estabelecer parâmetros de qualidade que possam ser percebidos tanto pelos hóspedes nacionais como pelos estrangeiros. As empresas hoteleiras que optam pela certificação (adesão voluntária) passam pela inspeção e avaliação feita pelo MTur em parceria com o Inmetro (Instituto Nacional de Metrologia, Qualidade e Tecnologia), nas quais precisam

1. SISTEMA BRASILEIRO DE CLASSIFICAÇÃO DOS MEIOS DE HOSPEDAGEM. Disponível em: <http:// www.classificacao.turismo.gov.br/MTUR-classificacao/mtur-site/ >. Acesso em: 5 de maio 2015. 
atender a uma série de requisitos mandatórios e eletivos relacionados à infraestrutura, aos serviços e à sustentabilidade. Assim, subentende-se que um hóspede, ao observar uma placa fixada na entrada de um hotel apresentando determinado número de estrelas, sente-se seguro de que a infraestrutura e a qualidade dos serviços prestados foram auditadas, e que o empreendimento, certificado pelo SBClass, atende a vários parâmetros de qualidade, podendo assim gerar elevada ou baixa expectativa de acordo com o número de estrelas atribuídas ao meio de hospedagem. Para Kotler (1999, p. 6), nessa situação o ideal seria encontrar "o equilíbrio entre as expectativas do cliente com o desempenho da empresa". Conforme o autor, as empresas precisam encantar os clientes, prometendo somente o que podem oferecer para depois ir além do que prometeram. 0 conceito de qualidade, em especial quando se refere a serviços, é totalmente subjetivo. Um serviço pode ser avaliado com base em diferentes dimensões (acesso, velocidade, competência, atendimento, flexibilidade, segurança, custos, comunicação, limpeza, conforto e qualidade dos bens e produtos) e o grau de importância dessas variáveis está associado às necessidades individuais do cliente (CARVALHO; PALADINI, 2012). A qualidade percebida não diz respeito à opinião do empreendedor em relação ao seu negócio nem à maneira de entregar com qualidade, ou mesmo o preço pago pelo consumidor. 0 que realmente vale é a percepção do cliente. Em concordância, Heckert e Silva (2001, p. 321) ressaltam que "a avaliação da qualidade depende muito mais dos valores atribuídos pelo cliente do que das características intrínsecas do produto ou serviço". Logo, é imprescindível descobrir, entre os vários atributos elencados, quais são os mais importantes/críticos na opinião dos consumidores (CASTELLI, 2001). Com a introdução e a intensa utilização das redes sociais, o acesso às percepções dos clientes sobre os serviços prestados pelas organizações ficou mais fácil e rápido. As redes sociais vêm se tornando o ambiente de conversação e troca de informações das mais diversas faixas etárias. Entre os assuntos variados, estão as sugestões e opiniões positivas ou negativas sobre produtos e serviços (RECUERO, 2009; SILVA, 2010). No caso das empresas hoteleiras, duas redes sociais merecem destaque: o TripAdvisor e o Booking. com. Ambas vêm sendo consideradas grandes termômetros para identificar e certificar os serviços prestados aos hóspedes. Por apresentar avaliações de clientes e divulgar as experiências vivenciadas durante as suas estadas, as referidas redes sociais se tornaram ferramentas de pesquisa para quem deseja viajar. Com base no exposto, esta pesquisa busca responder à seguinte questão: como os meios de hospedagem localizados na região Nordeste, classificados pelo SBClass nas categorias cinco e quatro estrelas, vêm sendo avaliados no TripAdvisor e no Booking.com? Para tanto, buscou-se identificar quais hotéis da região Nordeste foram classificados pelo SBClass nas categorias mencionadas e analisar, de forma comparativa, as notas atribuídas a esses meios de hospedagem e os comentários divulgados no TripAdvisor e no Booking.com.

\section{QUALIDADE DOS SERVIÇOS DE HOTELARIA}

A busca pela melhoria contínua por parte das organizações está associada ao crescimento do nível de exigência dos consumidores. Buscando um diferencial para se sobrepor às concorrentes, em um contexto marcado pela competitividade, as organizações passaram a se preocupar de maneira perspicaz e eficiente com a oferta de serviços de qualidade, vista como fator determinante para o su- 
cesso e a sustentabilidade organizacional. Também no setor hoteleiro a qualidade é considerada um elemento fundamental para satisfação do hóspede, cujo valor é primordial na hotelaria, visto que, caso tenha uma boa experiência no hotel em que está hospedado, não só poderá voltar ao local como possivelmente fará uma divulgação positiva do empreendimento para amigos e familiares (LAU; AKBAR; FIE, 2005). Nadiri e Hussain (2005) enfatizam que empreendimentos hoteleiros que prestam serviços de qualidade possuem uma viabilidade maior no mercado e, consequentemente, um crescimento da confiabilidade diante dos hóspedes. Desse modo, os gestores de meios de hospedagem têm que buscar maneiras de oferecer bens e serviços de qualidade, superando os seus concorrentes. Para tanto, faz-se necessário compreender e satisfazer às necessidades de seus clientes e ultrapassar suas expectativas.

Corrêa e Caon (2006) destacam a relação direta entre o grau de satisfação do cliente e a fidelização e retenção e, por conseguinte, a lucratividade da organização. Castelli (1994, p. 15-16) enfatiza: "investir em qualidade resulta em menos defeitos, produtos melhores, posição financeira melhor, maior bem-estar, menos giro de pessoal, menor absenteísmo, clientes satisfeitos e uma imagem melhor".

Entende-se por satisfação o "sentimento de prazer ou de desapontamento resultante da comparação do desempenho esperado pelo produto ou serviço (ou resultado) em relação às expectativas da pessoa" (KOTLER, 1998, p. 53). A expectativa do cliente tem sua formação decorrente de uma série de fatores, como a comunicação boca a boca, que sempre parte de uma experiência passada positiva ou negativa. Esse tipo de propaganda pode ascender ou demolir a reputação de uma empresa. Mesmo que nos serviços seja levado em consideração o lado subjetivo, ou seja, cada um tem sua visão do que é um bom serviço, os potenciais clientes se baseiam muito nas experiências passadas de outros clientes (CARVALHO; PALADINI, 2012; CORREA; CAON, 2006; GIANESI; CORREAA, 1994; GRÖNROOS, 1993; KOTLER, 1998). Além da comunicação boca a boca, outro fator que merece destaque na formação da expectativa é a comunicação externa, composta por vários tipos de comunicação, da própria organização ou de outros órgãos de divulgação. Carvalho e Paladini (2012) destacam que "a expectativa já vem com o cliente, mas pode ser incrementada pela empresa”, não podendo ser elevada ao ponto em que não possa ser atingida pelo prestador de serviço nem podendo ser tão baixa a ponto de não atrair o cliente. A chave para a satisfação, nesse sentido, é o equilibro entre as expectativas e o desempenho (KOTLER, 1999, p. 6). Entende-se por expectativa a referência utilizada pelo cliente para avaliar se o serviço é de boa qualidade, originada, geralmente, por uma promessa, um direito ou uma esperança que esse cliente possui, formando assim uma lacuna que necessita ser preenchida para sua plena satisfação (CARVALHO; PALADINI, 2012; HECKERT; SILVA, 2001). De acordo com Paladini (2000), a qualidade em serviços pode ser definida como o grau em que as expectativas do cliente são atendidas por sua percepção do serviço prestado, com base em vários critérios de avaliação. Consideram-se critérios de avaliação os aspectos que podem ser utilizados pelos consumidores para medir a qualidade dos serviços dos estabelecimentos, dentre os quais se destacam: acesso, velocidade, competência, atendimento, flexibilidade, segurança, custos, comunicação, limpeza, conforto e qualidade dos bens e produtos (CARVALHO; PALADINI, 2012; DOMINGUES, 1999; GARVIN, 2002), cujas definições podem ser visualizadas no Quadro 1. 
Quadro 1 - Critérios de avaliação da qualidade do serviço

\begin{tabular}{|c|c|}
\hline Critério & Definição \\
\hline Acesso & $\begin{array}{l}\text { Facilidade, rapidez, praticidade de se chegar ao estabelecimento ou ao local } \\
\text { onde o cliente vai se acomodar. }\end{array}$ \\
\hline Velocidade & $\begin{array}{l}\text { Rapidez entre a chegada do cliente ao estabelecimento e o atendimento e } \\
\text { entre o atendimento e a entrega do serviço. }\end{array}$ \\
\hline Competência & $\begin{array}{l}\text { Capacidade técnica que todos os membros da organização têm de } \\
\text { prestar o serviço. }\end{array}$ \\
\hline Atendimento & $\begin{array}{l}0 \text { auxílio dado pelos funcionários aos clientes, unido à simpatia e à cortesia } \\
\text { na forma de tratamento direto com o consumidor. }\end{array}$ \\
\hline Flexibilidade & $\begin{array}{l}\text { Capacidade de atender às necessidades e desejos dos clientes, por mais } \\
\text { variados que sejam, oferecendo várias opções de serviços e meios de } \\
\text { pagamento. }\end{array}$ \\
\hline Segurança & $\begin{array}{l}\text { Sentimento de proteção por parte do cliente, tanto pessoal como } \\
\text { dos seus bens. }\end{array}$ \\
\hline Custos & $\begin{array}{l}\text { Todas as possibilidades de gastos que os clientes possam perceber, } \\
\text { tanto com os preços dos serviços como com os custos para se chegar ao } \\
\text { estabelecimento. }\end{array}$ \\
\hline Comunicação & $\begin{array}{l}\text { A forma adequada do prestador do serviço comunicar-se com o cliente, de } \\
\text { modo que seja transmitido tudo aquilo que o consumidor necessita para } \\
\text { esclarecimento do serviço prestado. }\end{array}$ \\
\hline Limpeza & Higiene do estabelecimento, das instalações e dos equipamentos. \\
\hline Conforto & Comodidade ao cliente por meio de instalações, assentos etc. \\
\hline $\begin{array}{l}\text { Qualidade dos } \\
\text { bens e produtos }\end{array}$ & $\begin{array}{l}\text { Qualidade do material dos bens do estabelecimento e qualidade dos } \\
\text { produtos oferecidos, ingredientes etc. }\end{array}$ \\
\hline
\end{tabular}

Fonte - Adaptado de Carvalho e Paladini (2012, p. 340-341)

Entre esses critérios, no contexto da hotelaria, os mais utilizados são localização, atendimento/funcionários, relação custo-benefício e limpeza - critérios, esses, avaliados nas duas redes sociais analisadas nesta pesquisa.

\section{REDES SOCIAIS E HOTELARIA}

Diariamente, usuários da internet postam, publicam, criam, produzem e repassam informações sobre diversos assuntos, inclusive sobre suas satisfações ou insatisfações com os serviços prestados pelos meios de hospedagem. De acordo com Andrade e Ferreira (2012), a internet foi uma das maiores inovações do século passado, pois além de transformar a comunicação, modificou também a maneira dos indivíduos se relacionarem e realizarem operações financeiras e comerciais. Com essa produção de informação e intensa interação, torna-se complexo controlar tudo o que está sendo dito pelos usuários em todas as redes e gerenciar a imagem organizacional difundida na internet. No entanto, o monitoramento desses conteúdos passa a ser uma necessidade, para que organizações ou celebridades saibam o que está sendo dito a respeito de sua marca (SILVA, 2010). Nessa nova era, qualquer pessoa que tiver acesso à internet pode se comunicar com outra, não importando a localidade em que se encontra, tendo acesso a informações na mesma hora e participando de operações comerciais sem sair do lugar (ANDRADE; FERREIRA, 2012). No contexto da hotelaria, duas redes sociais vêm se destacando: TripAdvisor e Booking.com. 
Em ambas as ferramentas, os turistas podem buscar informações e comparar os preços e a qualidade dos serviços de meios de hospedagem de todos os lugares do mundo. Todavia, cada uma tem seu diferencial, e no Quadro 2 pode-se observar a comparação entre elas. Observa-se que, apesar de parecerem bem semelhantes, as duas redes possuem características singulares. De todo modo, ambas são eficientes no que se propõem e podem ser utilizadas como ferramentas complementares. 0 Booking.com e o TripAdvisor, cada dia mais utilizados, tornaram-se referências no segmento hoteleiro.

Quadro 2 - Principais diferenças entre o TripAdvisor e o Booking.com

\begin{tabular}{|l|l|l|}
\hline Critério & TripAdvisor & Booking.com \\
\hline \multirow{2}{*}{ Pesquisa } & $\begin{array}{l}\text { É mais completo: podem ser } \\
\text { encontradas informações não só de } \\
\text { meios de hospedagem, mas de outros } \\
\text { estabelecimentos e informações } \\
\text { turísticas de modo geral. }\end{array}$ & $\begin{array}{l}\text { É mais prático e simples: permite } \\
\text { visualizar os preços cobrados e } \\
\text { realizar reservas em meios de } \\
\text { hospedagem. }\end{array}$ \\
\hline \multirow{2}{*}{ Layout } & $\begin{array}{l}\text { Tem muitos conteúdos diferentes, o } \\
\text { que pode fazer que o usuário menos } \\
\text { acostumado com o site fique um pouco } \\
\text { confuso na navegação. }\end{array}$ & $\begin{array}{l}\text { É mais simples e organizado. Basta } \\
\text { inserir local e data, ver as opções } \\
\text { de meio de hospedagem e fazer a } \\
\text { reserva. }\end{array}$ \\
\hline
\end{tabular}

Fonte - Elaborado pelos autores (2015)

O TripAdvisor é conhecido mundialmente, recebe mais de sessenta milhões de visitantes por mês e cerca de cinquenta postagens por minuto. Com base em uma pesquisa interna do site, o viajante lê em média doze opiniões antes de efetuar sua reserva (REVISTA HOTÉIS, 2012). 0 Booking.com, por sua vez, possui atualmente 1.180.081 acomodações cadastradas, de diversos lugares do mundo e dispõe de 115.830.000 avaliações em sua página (BOOKING.COM, 2017). Em razão da representatividade das duas redes sociais para o segmento hoteleiro, ambas foram selecionadas como objeto de estudo. Na próxima seção, serão apresentados os procedimentos metodológicos utilizados para a realização desta pesquisa.

\section{PROCEDIMENTOS METODOLÓGICOS}

Buscando atingir o objetivo central deste trabalho, que consiste em analisar a percepção de hóspedes sobre a qualidade dos serviços de empresas hoteleiras classificadas pelo SBClass nas categorias cinco e quatro estrelas a partir de avaliações divulgadas no TripAdvisor e no Booking.com, realizou-se uma pesquisa descritiva de abordagem qualitativa. A escolha pela abordagem qualitativa foi motivada pela necessidade de se responder à questão central da pesquisa por meio de um processo de interpretação. 0 trabalho realizado se caracteriza como um estudo descritivo por buscar especificar e caracterizar a percepção de um grupo de pessoas sobre o objeto que está sob análise (DENCKER, 1998; GIL, 2008; HAIR JÚNIOR et al., 2005; SAMPIERI; COLLADO; LUCIO, 1991). Quanto ao contexto e aos sujeitos da pesquisa, teve como objeto todos os meios de hospedagem classificados pelo SBClass nas categorias de cinco e quatro estrelas da região Nordeste. 0 universo compreenderia, portanto, nove empresas hoteleiras. Entretanto, uma delas, a União Empreendimentos Turísticos Ltda - Aracajú/ SE, foi descartada por não ter sido encontrada no TripAdvisor e no Booking.com, possivelmente em razão de o nome jurídico não corresponder ao nome fantasia 
utilizado pelo hotel, o que pode ter impedido sua identificação e localização nas redes sociais. 0 outro meio de hospedagem descartado foi o Matiz Salvador, por não constar no TripAdvisor. Dessa forma, a análise se restringiu a sete empreendimentos hoteleiros: quatro resorts e três hotéis. Todos serão tratados nesta pesquisa com a abreviação "MH" (meio de hospedagem) seguida por um número, como pode ser observado no Quadro 3. Os sujeitos da pesquisa foram os usuários das duas redes em análise que avaliaram os meios de hospedagem em estudo, seja comentaram ou atribuindo notas a eles.

Quadro 3 - Relação dos meios de hospedagem localizados na região nordeste que foram objetos de análise desse estudo

\begin{tabular}{|l|l|c|}
$\begin{array}{l}\text { Classificação pelo } \\
\text { SBClass }\end{array}$ & Meio de hospedagem & Código \\
\hline & $\begin{array}{l}\text { Vila Galé Marés - Monte Gordo/BA (resort) } \\
\text { Vila Galé Cumbuco - Caucaia/CE (resort) }\end{array}$ & MH 1 \\
\hline \multirow{2}{*}{5 estrelas } & $\begin{array}{l}\text { Ocean Palace Beach Resorts e Bungalows - } \\
\text { - Natal/RN (resort) }\end{array}$ & MH 3 \\
\hline & SERHS Natal Grand Hotel - Natal/RN (resort) & MH 4 \\
\hline \multirow{2}{*}{4 estrelas } & Hotel Vila do Mar - Natal/RN (hotel) & MH 5 \\
\hline & Aquários Praia Hotel - Aracajú/SE (hotel) & MH 6 \\
\hline & Celi Praia Hotel - Aracajú/SE (hotel) & MH 7 \\
\hline
\end{tabular}

Fonte - Elaborado pelos autores (2015)

Depois da identificação dos meios de hospedagem por meio de informações do SBClass divulgadas no site do MTur, foram analisadas as notas atribuídas a cada um dos estabelecimentos no Booking.com e no TripAdvisor, bem como as pontuações obtidas nos critérios "localização", "atendimento/funcionários", "custo-benefício" e "limpeza". Em seguida, foram analisados os cinco últimos comentários postados tanto no Booking.com como no TripAdvisor, presentes em suas respectivas páginas no dia 12 de maio de 2015.

\section{RESULTADOS}

Na Tabela 1 podem ser visualizadas as notas obtidas pelos sete meios de hospedagem analisados no site TripAdvisor. Sabendo-se que os quatro primeiros foram classificados pelo SBClass com cinco estrelas, e os três últimos com quatro estrelas, pode-se constatar que não houve diferença significativa em relação às avaliações observadas de acordo com os critérios do TripAdvisor.

Tabela 1 - Avaliações dos meios de hospedagem no TripAdvisor

\begin{tabular}{|l|l|l|l|l|l|l|l|}
\hline Hotel/Nota nos critérios & MH 1 & MH 2 & MH 3 & MH 4 & MH 5 & MH 6 & MH 7 \\
\hline Localização & 9,0 & 8,0 & 9,0 & 9,0 & 8,0 & 9,0 & 9,0 \\
\hline Atendimento/funcionários & 7,0 & 8,0 & 8,0 & 9,0 & 8,0 & 8,0 & 9,0 \\
\hline Custo-benefício & 7,0 & 7,0 & 8,0 & 8,0 & 7,0 & 7,0 & 8,0 \\
\hline Limpeza & 8,0 & 8,0 & 8,0 & 9,0 & 8,0 & 8,0 & 9,0 \\
\hline
\end{tabular}

Fonte - Elaborada pelos autores (2015) 
Verifica-se na Tabela 1 que as notas obtidas no critério "localização" não apresentaram grande variação. Já no critério "atendimento/funcionários", o MH 1, cuja classificação é de cinco estrelas, apresentou a menor nota, enquanto MH 7, de classificação quatro estrelas, recebeu a nota 9,0. 0 critério "custo-benefício" foi o que apresentou as menores notas em todos os meios de hospedagem analisados, variando entre 7,0 e 8,0. Já no critério "limpeza", apenas dois empreendimentos hoteleiros receberam a nota 9,0; os demais receberam 8,0. Na Tabela 2, podem ser analisadas as notas obtidas pelos sete meios de hospedagem avaliados no site Booking.com. Pode-se observar que as notas atribuídas tanto aos hotéis de cinco estrelas quanto aos hotéis de quatro estrelas não apresentam variação significativa, não sendo possível afirmar, somente com base nas notas, se os hotéis analisados estão melhor ou pior classificados no SBClass.

Tabela 2 - Avaliações dos meios de hospedagem no Booking.com

\begin{tabular}{|l|c|c|c|c|c|c|c|}
\hline Hotel/Nota nos critérios & MH 1 & MH 2 & MH 3 & MH 4 & MH 5 & MH 6 & MH 7 \\
\hline Localização & 8,6 & 8,1 & 8,9 & 9,0 & 8,3 & 9,1 & 9,1 \\
\hline Atendimento/funcionários & 7,3 & 7,8 & 7,9 & 8,8 & 7,6 & 7,7 & 8,8 \\
\hline Custo-benefício & 7,1 & 7,2 & 7,1 & 7,7 & 7,3 & 7,2 & 8,0 \\
\hline Limpeza & 7,5 & 7,8 & 7,9 & 8,6 & 7,7 & 7,9 & 8,8 \\
\hline
\end{tabular}

Fonte - Elaborada pelos autores (2015)

Em vários dos critérios destacados, meios de hospedagem classificados com quatro estrelas receberam notas superiores àquelas recebidas pelos empreendimentos classificados com cinco estrelas, como no caso do critério "localização", em que os meios de hospedagem 6 e 7 receberam as melhores avaliações. No critério "atendimento/funcionários", ocorre situação semelhante, em que a melhor nota se repete para o MH 4 (cinco estrelas) e o MH 7 (quatro estrelas), enquanto a pior nota é atribuída ao MH 1 (cinco estrelas). No critério "custo-benefício", as duas piores notas também foram atribuídas a meios de hospedagem classificados com cinco estrelas. Já no critério "limpeza”, a melhor nota foi atribuída ao $\mathrm{MH} \mathrm{7,} \mathrm{de} \mathrm{quatro} \mathrm{estrelas.} \mathrm{A} \mathrm{seguir,} \mathrm{serão} \mathrm{apresentados}$ os resultados obtidos por meio da análise dos cinco últimos comentários de clientes nos sites do TripAdvisor e Booking.com. No Quadro 4, pode-se visualizar os comentários relativos ao MH 1.

Quadro 4 - Análise comparativa dos comentários relacionados ao MH 1

(Vila Galé Marés) divulgados nas duas redes sociais

\section{Comentários positivos}

- "Ideal para viagens com crianças recreação muito boa" (TripAdvisor);

- "Ambiente agradável e piscinas maravilhosas" (TripAdvisor);

- "O hotel é fantástico e o atendimento espetacular" (Booking.com);

- "Tudo maravilhoso" (Booking.com).

\section{Comentários negativos}

- "Atendimento/funcionários pouco prestativos";

- "Foi uma decepção: manutenção ruim, enxoval velho, restaurante quente, comida ruim";

- "Hotel caro para o que oferece";

- "Achei que para receber o nome de resorts falta muito" (Booking.com).

Fonte - Elaborado pelos autores (2015) 
A seguir, no Quadro 5, podem ser verificados os comentários mais relevantes sobre o MH 2. Observa-se que o bom atendimento e a atenção dos funcionários aos clientes foram mencionados três vezes como uma qualidade do meio de hospedagem, além do fato de haver piscinas, os quartos e a alimentação, que também foram citados três vezes como aspectos positivos do hotel no Booking.com.

Quadro 5 - Análise comparativa dos comentários relacionados ao MH 2

(Vila Galé Cumbuco) divulgados nas duas redes sociais

\begin{tabular}{|c|c|}
\hline Comentários positivos & Comentários negativos \\
\hline $\begin{array}{l}\text { - "Gastronomia farta" (TripAdvisor); } \\
\text { - Animadores disponíveis e cuidadosos } \\
\text { (TripAdvisor); } \\
\text { - Excelente atendimento - "Sempre } \\
\text { prontos a nos servirem" (TripAdvisor); } \\
\text { - "Refeições variadas e deliciosas" } \\
\text { (TripAdvisor); } \\
\text { - Funcionários atenciosos (TripAdvisor); }\end{array}$ & $\begin{array}{l}\text { - Check-in muito lento (TripAdvisor); } \\
\text { - Não há Wi-Fi nos quartos (TripAdvisor); } \\
\text { - "Não é um bom resort. Chopp Kaiser, Cerveja } \\
\text { Bavária, falta de variedade nas refeições, falta } \\
\text { de cortesia dos funcionários da recepção, não } \\
\text { há Wi-Fi” (TripAdvisor); } \\
\text { - Preços abusivos pelos serviços de fotografia } \\
\text { - } \text { dentro do hotel (TripAdvisor); } \\
\text { (Booking.com); } \\
\text { - "Recepção com atendimento precário, } \\
\text { péssimo, inacreditável que uma estrutura } \\
\text { como esta possa não ter uma mão de obra } \\
\text { adequada" (Booking.com). }\end{array}$ \\
\hline
\end{tabular}

Fonte - Elaborado pelos autores (2015)

Por outro lado, nos comentários negativos é possível constatar que o atendimento dos funcionários da recepção foi considerado ruim por duas vezes, com um dos clientes chegando a qualificar o serviço como "precário, péssimo e inacreditável que uma estrutura como esta possa não ter uma mão de obra adequada".

No Quadro 6, pode-se observar os comentários contidos nas avaliações do MH 3. Verifica-se que o meio de hospedagem recebeu vários elogios em relação ao atendimento, café da manhã, localização e piscinas, tendo sido citado por dois dos clientes como "hotel maravilhoso".

Quadro 6 - Análise comparativa dos comentários relacionados ao MH 3 (Ocean Palace Beach Resort \& Bungalows) divulgados nas duas redes sociais

\begin{tabular}{|c|c|}
\hline Comentários positivos & Comentários negativos \\
\hline $\begin{array}{l}\text { - “Ótimo atendimento, café da manhã muito } \\
\text { farto, muitas piscinas, quartos amplos" } \\
\text { (TripAdvisor); } \\
\text { - "Café da manhã inesquecível, excelente } \\
\text { atendimento por todos os funcionários, } \\
\text { uma piscina melhor que a outra, tudo } \\
\text { muito organizado e muito limpo [...] } \\
\text { Esse lugar é maravilhoso" (TripAdvisor); } \\
\text { - "Ótima cama” (TripAdvisor); } \\
\text { - "Linda localização". (TripAdvisor); } \\
\text { - "Hotel maravilhoso" (Booking.com); } \\
\text { - "Excepcional” (Booking.com). }\end{array}$ & $\begin{array}{l}\text { - Refeições muito caras dentro do hotel } \\
\text { (TripAdvisor); } \\
\text { - Estacionamento insuficiente (TripAdvisor); } \\
\text { - Atendimento "impessoal" (Booking.com); } \\
\text { - Manutenção ruim, preços das bebidas caros } \\
\text { (Booking.com); } \\
\text { - Restaurantes caros - "Fora da realidade"; } \\
\text { manutenção ruim, elevadores e chuveiro } \\
\text { sem funcionar (Booking.com). }\end{array}$ \\
\hline
\end{tabular}

Fonte - Elaborado pelos autores (2015) 
Em relação aos comentários negativos, ainda relacionados ao $\mathrm{MH}$ 3, destacam-se os que não ficaram satisfeitos com os preços cobrados nos restaurantes desse meio de hospedagem, com um dos clientes chegando a classificar esses preços como "fora da realidade".

No Quadro 7, é possível visualizar uma mostra dos comentários dos clientes do $\mathrm{MH} 4$.

Quadro 7 - Análise comparativa dos comentários relacionados ao MH 4 (SERHS Natal Grand Hotel) divulgados nas duas redes sociais

\begin{tabular}{l|l}
\hline Comentários positivos & Comentários negativos \\
\hline - Quarto, café da manhã e atendimento ótimos & \\
$\begin{array}{l}\text { (TripAdvisor); } \\
\text { - "O atendimento de todos os funcionários foi } \\
\text { muito bom" (TripAdvisor); }\end{array}$ & $\begin{array}{l}\text { - "Comida muito cara, recomendo comer } \\
\text { fora" (TripAdvisor); }\end{array}$ \\
- Atendimento, quartos e recreação muito bons & - Piscina aquecida não funcionou \\
(TripAdvisor); & (Booking.com); \\
- Atendimento, café da manhã, limpeza dos & - Atendimento bar piscina ruim - poucos \\
$\begin{array}{l}\text { quartos, piscinas e recreação muito bons } \\
\text { (TripAdvisor); }\end{array}$ & atendentes (Booking.com); \\
- "Excelente localização, bons restaurantes & Café da manhã com muita gente \\
internos e equipe muito atenciosa" & - Restaurante sem diferencial e quarto \\
(Booking.com); & barulhento (Booking.com); \\
Equipe atenciosa e "café da manhã & \\
excepcional" (Booking.com). &
\end{tabular}

Fonte - Elaborado pelos autores (2015)

Como pode ser observado no Quadro 7, o atendimento foi considerado pelos hóspedes como excelente. Em contrapartida, verifica-se que o meio de hospedagem recebeu queixas diversificadas e sem repetições, o que pode representar falhas isoladas do hotel, como no café da manhã, por exemplo, ou na piscina aquecida, que não estava na temperatura ideal para o hóspede.

No Quadro 8, é possível observar os comentários mais relevantes em relação ao MH 5.

Quadro 8 - Análise comparativa dos comentários relacionados ao MH 5 (Hotel Vila do Mar) divulgados nas duas redes sociais

\begin{tabular}{|c|c|}
\hline Comentários positivos & Comentários negativos \\
\hline $\begin{array}{l}\text { - "Quartos espaçosos, piscina maravilhosa, } \\
\text { café da manhã muito bom" (TripAdvisor); } \\
\text { - "Atendimento atencioso, limpeza muito boa } \\
\text { e localização excelente" (TripAdvisor); } \\
\text { - "Atendimento, manutenção e quartos } \\
\text { ótimos", "a modernidade do hotel me } \\
\text { surpreendeu" (TripAdvisor); } \\
\text { - Quartos confortáveis e boa comida } \\
\text { (TripAdvisor). }\end{array}$ & $\begin{array}{l}\text { - "Check-in muito tarde" (TripAdvisor); } \\
\text { - "Sem Wi-Fi nos quartos e cama sem } \\
\text { conforto (TripAdvisor); } \\
\text { - } \text { Recepção e piscinas longes dos quartos } \\
\text { (TripAdvisor); } \\
\text { - Wi-Fi ruim (BOOKING.COM); } \\
\text { - Ar condicionado antigo e barulhento } \\
\text { (Booking.com); } \\
\text { - Check-in demorado (Booking.com); } \\
\text { - Ar-condicionado ruim (Booking.com). }\end{array}$ \\
\hline
\end{tabular}

Fonte - Elaborado pelos autores (2015)

Vê-se que os quartos foram citados como ótimos e espaçosos por dois clientes; como aspecto negativo, a má qualidade da internet sem fio foi citada por 
três clientes; em outros dois comentários, é citada também a insatisfação com o check-in.

No Quadro 9, constam os principais comentários em relação ao MH 6.

Quadro 9 - Análise comparativa dos comentários relacionados ao MH 6 (Aquários Praia Hotel) divulgados nas duas redes sociais

\begin{tabular}{|c|c|}
\hline Comentários positivos & Comentários negativos \\
\hline $\begin{array}{l}\text { - Localização foi citada quatro vezes como } \\
\text { aspecto positivo do hotel (TripAdvisor); } \\
\text { - Atendimento foi citado duas vezes como } \\
\text { aspecto positivo do hotel (TripAdvisor); } \\
\text { - Localização foi citada três vezes como } \\
\text { aspecto positivo do hotel (Booking.com); } \\
\text { - A cama foi elogiada uma vez (Booking.com). }\end{array}$ & $\begin{array}{l}\text { - Cortina no box do banheiro foi citado duas } \\
\text { vezes como aspecto negativo do hotel } \\
\text { (TripAdvisor); } \\
\text { - Falta de estacionamento foi citado três } \\
\text { vezes como aspecto negativo do hotel } \\
\text { (TripAdvisor); } \\
\text { - "Hotel um pouco velho" (Booking.com); } \\
\text { - Cortina no box do banheiro foi citado } \\
\text { novamente como aspecto negativo } \\
\text { (Booking.com); } \\
\text { "Quarto sujo e mal lavado. Fiquei } \\
\text { decepcionado" (Booking.com). }\end{array}$ \\
\hline
\end{tabular}

Fonte - Elaborado pelos autores (2015)

Dos dez comentários analisados, a excelente localização foi citada em sete, o que representa um aspecto bastante relevante desse meio de hospedagem. Entre os comentários negativos, destacam-se o uso de cortina no box dos banheiros e a falta de estacionamento, ambos tendo sido citados duas vezes pelos hóspedes.

No Quadro 10, é possível observar os comentários mais relevantes referentes ao MH 7.

Quadro 10 - Análise comparativa dos comentários relacionados ao MH 7

(Celi Praia Hotel) divulgados nas duas redes sociais

\section{Comentários positivos}

- "Localização foi citada três vezes como aspecto positivo do hotel" (TripAdvisor);

- Atendimento foi citado duas vezes como aspecto positivo do hotel (TripAdvisor);

- Quarto confortável foi citado quatro vezes como aspecto positivo do hotel (TripAdvisor);

- Preços do restaurante justos (TripAdvisor);

- "Localização foi citada duas vezes como aspecto positivo do hotel" (Booking.com);

- Atendimento foi citado três vezes como aspecto positivo do hotel (Booking.com).

\section{Comentários negativos}

- Wi-fi ruim (TripAdvisor);

- Estacionamento foi citado duas vezes como aspecto ruim do hotel (TripAdvisor);

- "Check-in demorado e muito burocrático" (TripAdvisor);

- Wi-Fi ruim, falta d'água e arcondicionado ruim (Booking.com);

- Elevador com cartão (Booking.com).

Fonte - Elaborado pelos autores (2015)

De maneira semelhante ao MH 6, a localização do MH 7 também foi considerada diversas vezes (cinco comentários) como excelente. Já a falta de estacionamento foi citada duas vezes como aspecto negativo. Também o sinal da internet sem fio no hotel foi mencionado por duas vezes como ruim.

Na Tabela 3, é possível observar as notas gerais dos sete meios de hospedagem estudados nesta pesquisa, tanto no TripAdvisor como no Booking.com. Também foram calculadas as médias das notas e destacadas as melhores e piores notas observadas nos comentários de cada um dos sites. 
Pode-se destacar, em relação aos dados da Tabela 3, que o MH 1 recebeu 2,0 de um de seus clientes, a pior nota observada, enquanto outros clientes lhe atribuíram nota 10. Essa alta variação de nota pode ser justificada pelo fato de a hotelaria se enquadrar no setor de prestadores de serviços.

Tabela 3 - Notas atribuídas aos meios de hospedagem no TripAdvisor e no Booking.com

\begin{tabular}{|l|c|c|c|c|c|c|c|}
\hline Hotel/Nota nos critérios & MH 1 & MH 2 & MH 3 & MH 4 & MH 5 & MH 6 & MH 7 \\
\hline \begin{tabular}{l|l|l|l|l|} 
Nota geral no TripAdvisor \\
Nota geral no Booking.com
\end{tabular} & 7,0 & 8,0 & 9,0 & 9,0 & 8,0 & 8,0 & 9,0 \\
\hline $\begin{array}{l}\text { Média das notas dos cinco } \\
\text { comentários - TripAdvisor }\end{array}$ & 6,4 & 8,0 & 8,4 & 10,0 & 8,4 & 8,0 & 7,2 \\
\hline $\begin{array}{l}\text { Média das notas dos cinco } \\
\text { comentários - Booking.com }\end{array}$ & 8,4 & 7,7 & 8,4 & 8,9 & 8,8 & 7,0 & 8,4 \\
\hline $\begin{array}{l}\text { Melhor nota dos comentários no } \\
\text { Booking.com }\end{array}$ & 10,0 & 9,5 & 10 & 10,0 & 10,0 & 10,0 & 9,6 \\
\hline $\begin{array}{l}\text { Pior nota dos comentários no } \\
\text { Booking.com }\end{array}$ & 5,4 & 6,7 & 5,8 & 7,1 & 7,9 & 6,0 & 5,8 \\
\hline $\begin{array}{l}\text { Melhor nota dos comentários no } \\
\text { TripAdvisor }\end{array}$ & 10,0 & 10,0 & 10,0 & 10,0 & 10,0 & 10,0 & 8,0 \\
\hline $\begin{array}{l}\text { Pior nota dos comentários no } \\
\text { TripAdvisor }\end{array}$ & 2,0 & 6,0 & 6,0 & - & 6,0 & 4,6 & 6,0 \\
\hline
\end{tabular}

Fonte - Elaborado pelos autores (2015)

Sendo assim, a característica de variabilidade se faz muito presente e expressiva, já que os serviços variam tanto em relação às pessoas que os prestam quanto às pessoas que os consomem. Além disso, qualidade é uma variável totalmente subjetiva, associada às necessidades individuais de casa pessoa. Outra informação relevante é que os cinco comentários capturados no TripAdvisor referentes ao MH 4 receberam nota 10; por isso, não houve "pior nota" para esse hotel. Observou-se ainda que todas as notas gerais do TripAdvisor são maiores do que as notas gerais atribuídas pelos clientes no Booking.com. 0 fato de que, no Booking. $\mathrm{com}$, apenas os clientes que de fato utilizaram os serviços do meio de hospedagem possam fazer avaliações pode ser uma possível explicação para a diferença. Também é relevante destacar que não se observou variedade significativa entre as notas recebidas pelos quatro primeiros meios de hospedagem, classificados pelo SBClass com cinco estrelas, e os três últimos, classificados com quatro estrelas. Como exemplo, podemos citar o $\mathrm{MH} 7$, com quatro estrelas de acordo com o SBClass, mas que possui notas gerais melhores que todos os outros hotéis de cinco estrelas, tanto no TripAdvisor como no Booking.com.

\section{CONSIDERAÇÕES FINAIS}

Com este estudo, ficou evidente que o termo "qualidade" não possui um conceito unificado que seja aplicável a qualquer segmento. Ao contrário, admitem-se várias abordagens com conceitos flexíveis e adaptáveis, pelo fato de que cada indivíduo desenvolve critérios que se unem para formar sua própria concepção sobre o nível de qualidade do objeto em análise com base na adequação às suas expectativas.

Além disso, destacou-se que o grau de satisfação do cliente é construído a partir da relação entre expectativa e experiência, entendida a expectativa como 
referência utilizada pelo cliente para avaliar se o serviço é de boa qualidade. Essa expectativa é originada, geralmente, por uma promessa, um direito ou uma esperança que o cliente possui, formando assim uma lacuna que necessita ser preenchida para sua plena satisfação (CARVALHO; PALADINI, 2012; HECKERT; SILVA, 2001).

No contexto da hotelaria, um dos elementos que exercem influência na criação das expectativas dos clientes é a classificação dos meios de hospedagem certificada pelo SBClass. 0 número de estrelas fixadas na fachada de um hotel gera nos clientes uma expectativa sobre a qualidade dos serviços ofertados, o que fez emergir o interesse por essa pesquisa. Buscando analisar a percepção de hóspedes sobre os serviços de meios de hospedagem classificados pelo SBClass nas categorias de cinco e quatro estrelas, a partir de avaliações divulgadas no TripAdvisor e no Booking.com, verificou-se que não houve diferença relevante entre as notas recebidas pelos quatro resorts classificados pelo SBClass como cinco estrelas e os três hotéis classificados como de quatro estrelas.

Assim, comparando as avaliações obtidas pela média de notas atribuídas aos meios de hospedagem nos sites TripAdvisor e Booking.com com as categorias em que esses mesmos empreendimentos foram classificados pelo SBClass, verificou-se que há certa incoerência, pois, se um cliente optar por escolher o hotel em que se hospedará por meio de uma análise das notas obtidas pelos meios de hospedagem no TripAdvisor e no Booking.com, ele pode optar por um de quatro estrelas em detrimento de um de cinco estrelas, tendo em vista que o primeiro terá recebido nota superior. Há uma grande variação entre as notas atribuídas pelos clientes nos comentários deixados no TripAdvisor e no Booking.com. 0 mesmo hotel pode receber a nota 10 de um cliente enquanto outro lhe atribui a nota 2 . Ou seja, enquanto um cliente se diz maravilhado com o hotel, outro se diz decepcionado e frustrado. Dessa forma, constatou-se que a característica de variabilidade dos serviços se faz muito presente e expressiva, pois os serviços dependem e variam tanto em relação às pessoas que os prestam como também em relação às pessoas que os recebem, além de, como já citado, qualidade ser uma variável totalmente subjetiva, associada às necessidades individuais de casa pessoa.

Este estudo é um esforço inicial na compreensão do processo de avaliação da qualidade dos serviços de hospedagem, fazendo-se necessária a realização de outras pesquisas que complementem e aprofundem a exploração da temática.

\section{REFERÊNCIAS}

ANDRADE, D. A. C.; FERREIRA, L. B. Compras coletivas na hotelaria: uma análise do ponto de vista do anunciante. In: SEMINÁRIO DA ASSOCIAÇÃO NACIONAL PESQUISA E PÓS-GRADUAÇÃO EM TURISMO, 9., 2012, São Paulo. Anais... São Paulo: Universidade Anhembi Morumbi, 2012.

B00KING.COM. 2017. Disponível em: <http://bit.ly/2nPa2oX>. Acesso em: 21 mar. 2017.

CARVALHO, M. M; PALADINI, E. P. Gestão da qualidade: teoria e casos. 2. ed. Rio de Janeiro: Elsevier, 2012.

CASTELLI, G. Excelência em hotelaria: uma abordagem prática. Rio de Janeiro: Qualitymark, 1994.

. Administração hoteleira. Caxias do Sul: Educs, 2001. 
CORRÊA, H. L.; CAON, M. Gestão de serviços: lucratividade por meio de operações e de satisfação dos clientes. São Paulo: Atlas, 2006.

DENCKER, A. F. M. Pesquisa em turismo: planejamento, métodos e técnicas. 9. ed. São Paulo: Futura, 1998.

DOMINGUES, M. E. M. Gestão da qualidade nos serviços hospitalares: estudos de caso. 172 p. Dissertação (Mestrado em Engenharia de Produção) - Centro de Ciências Exatas e de Tecnologia, Universidade Federal de São Carlos, São Carlos, 1999.

GARVIN, D. Gerenciando a qualidade. São Paulo. Qualitymark, 2002.

GIANESI, I. G. N.; CORRÊA, H. L. Administração estratégica de serviços: operações para satisfação do cliente. São Paulo: Atlas, 1994.

GIL, A. C. Métodos e técnicas de pesquisa social. 6. ed. São Paulo: Atlas, 2008.

GRÖNROOS, C. Marketing, gerenciamento e serviços: a competição por servir na Hora da Verdade. 6. ed. Rio de Janeiro: Campus, 1993.

HAIR JÚNIOR, J. F. et al. Fundamentos de métodos de pesquisa em administração. Porto Alegre: Bookman, 2005.

HECKERT, C. R.; SILVA, M. T. Qualidade de serviços nas organizações do terceiro setor. In: ENCONTRO NACIONAL DE ENGENHARIA DE PRODUÇÃO, 21., 2001, Salvador. Anais... Salvador, 2001.

KOTLER, P. Administração de marketing. 5. ed. São Paulo: Atlas, 1998.

. Marketing para o século XXI: como criar, conquistar e dominar mercados. São Paulo: Futura, 1999.

LAU, P. M.; AKBAR, A. K.; FIE, D. Y. G. Service quality: a study of the luxury hotel in Malaysia. Journal of American Academy of Business, Cambridge, v. 7, n. 2, p. 46-55, 2005.

NADIRI, H.; HUSSAIN, K. Perceptions of service quality in north Cyprus hotels. International Journal of Contemporary Hospitality Management, Bradford, v. 17, n. 6, p. 469-480, 2005.

PALADINI, E. P. Gestão da qualidade: teoria e prática. São Paulo: Atlas, 2000.

RECUERO, R. Redes sociais na internet. Porto Alegre: Salina, 2009.

REVISTA HOTÉIS. TripAdvisor ou SBClass: qual é a melhor classificação de um hotel? 2012. Disponível em: <http://www.revistahoteis.com.br/tripadvisor-ou-sbclass-qual-e-a-melhor-classificacao-de-um-hotel/>. Acesso em: 5 maio 2015.

SAMPIERI, R. H.; COLLADO, C. F.; LUCIO, B. P. Metodología de la investigación. México: Mcgraw Hill, 1991.

SILVA, T. Monitoramento de marcas e conversações. In: DOURADO, D. et al. (Org.). Mídias sociais: perspectivas, tendências e reflexões. Paper Cliq, 2010. Capítulo 41. Disponível em: <http://www.institutoconscienciago.com.br/pdf/ebookfinal-midias-sociais.pdf>. Acesso em: 10 mar. 2017.

Recebido em: 29/08/2016

Aprovado em: 19/01/2017 